\title{
SENSIBILIDADE DE FRANKLINIELLA SCHULTZEI (THYSANOPTERA: THRIPIDAE) A SOLVENTES
}

\author{
Vando Miossi Rondelli ${ }^{1}$ \\ Talita Cattem Mainetti Vargas ${ }^{2}$ \\ Davi Cardoso Aguiar de Melo ${ }^{3}$ \\ Wilker Pinheiro Lima ${ }^{4}$ \\ Wilson Rodrigues Valbon ${ }^{5}$ \\ Nayara Camatta Campos ${ }^{6}$ \\ Wenderson Tinorio de Paula ${ }^{7}$ \\ Dirceu Pratissoli ${ }^{8}$ \\ Adilson Vidal Costa ${ }^{9}$ \\ Vagner Tebaldi de Queiroz ${ }^{10}$
}

Resumo: O Brasil é um dos dez maiores produtores mundiais de tomate, com produção que atinge 3,7 milhões de toneladas. O tripes Frankliniella schultzei (Trybom) (Thysanoptera: Thripidae) mais comumente chamada de tripes-do-tomateiro, é umas das principais pragas desta cultura de grande importância econômica, onde os principais danos causados por essas pragas estão relacionados à sucção de seiva $e$ a transmissão de fitoviroses, as quais interferem no desenvolvimento das plantas. Com a finalidade de verificar a sensibilidade de $F$. schultzei frente a diferentes solventes, para serem utilizados no preparo de formulações contendo óleos essenciais, foram preparados e avaliados onze solventes dentre os mais utilizados em ensaios com insetos em concentrações variadas. Os resultados encontrados mostraram que cinco solventes: Tween $80^{\circledR}(0,05 \%)$, acetona (2\%), acetona (2\%)/Tween $80^{\circledR}(0,01 \%)$, DMSO (2\%), álcool $(2 \%)$, não diferiram estatisticamente quando comparados a água destilada. $O$ solvente escolhido para $O$ preparo das formulações foi acetona (2\%)/Tween $80^{\circledR}(0,01 \%)$ por apresentar melhor solubilidade frente ao óleo testado e causar baixa mortalidade frente ao tripes $(7,1 \%)$.

Palavras-chave: Tripes; Testemunha; Potencial inseticida; Testes biológicos.

\footnotetext{
1 Pesquisador/Universidade Federal de Rondônia. Brasil. E-mail: rondellimiossi@hotmail.com.

2 Graduada/Centro Universitário São Camilo, Brasil. E-mail: talitacattem@gmail.com.

${ }^{3}$ Graduado/Centro Universitário São Camilo, Brasil. E-mail: dcam21@hotmail.com.

4 Mestrando/Universidade Federal do Espírito Santo, Brasil. E-mail: wilker_plima@hotmail.com.

${ }^{5}$ Agronomia/Universidade Federal do Espírito Santo, Brasil. E-mail: wilson.valbon@gmail.com.

${ }^{6}$ Veterinária/ Universidade Federal do Espírito Santo, Brasil. E-mail: ncamattavet@gmail.com.

7 Química licenciatura/ Universidade Federal do Espírito Santo, Brasil, E-mail: wendersontinorio@hotmail.com.

8 Pesquisador/Universidade Federal do Espírito Santo, Brasil. E-mail: dirceu.pratissoli@gmail.com.

9 Pesquisador/Universidade Federal do Espírito Santo, Brasil. E-mail: avcosta@hotmail.com.

10 Orientador/Universidade Federal do Espírito Santo, Brasil. E-mail: vagnertq@gmail.com.
} 